\title{
A Cross Sectional Assessment on Distribution of Snail and Risk Factors of Schistosoma Mansoni Infection in Proximity to Water Contact Points in Gorgora Town, Western Dembia District, Northwest Ethiopia.
}

\author{
Aberham Abere \\ University of Gondar College of Medicine and Health Sciences \\ Ligabaw Worku \\ University of Gondar College of Medicine and Health Sciences \\ Abebe Genetu \\ AHRI: Armauer Hansen Research Institute \\ Dessie Abebaw \\ University of Gondar College of Medicine and Health Sciences \\ Adane Derso \\ University of Gondar College of Medicine and Health Sciences \\ Mulugeta Aemero ( $\boldsymbol{D}$ bmeskel@gmail.com ) \\ University of Gondar
}

\section{Research}

Keywords: Distribution, Snails, Schistosomiasis, Gorgora, Northwest Ethiopia

Posted Date: December 8th, 2020

DOl: https://doi.org/10.21203/rs.3.rs-117767/v1

License: (c) (i) This work is licensed under a Creative Commons Attribution 4.0 International License.

Read Full License 


\section{Abstract}

Background: Schistosomiasis is one of the great neglected tropical diseases with socio-economic and health problem worldwide. In Ethiopia numerous studies reported $S$. mansoni is high among school-age and preschool age children. Though intensive prevalence studies are conducted the snail distribution and infectivity status and human infection rate dynamics in Ethiopian context remains poorly understood. Thus the purpose of the current study was to assess distribution of snail and risk factors of Schistosoma mansoni infection in proximity to water contact points in Gorgora town, Western Dembia District, Northwest Ethiopia.

Method: A Community based cross-sectional study was conducted in Gorgora Town from March to May 2020. A total of 385 study participants were selected by systematic random sampling technique. Stool sample was collected and examined by Kato-Katz technique. Malacological survey was done from sampling sites showing signs of human activity near Lake Tana shoreline. Live snails collected in plastic bucket containing water and weed were transported within four hours to the University of Gondar, Medical parasitology laboratory for identification and determination of infection. Data was entered with EPI Data version 4.4.2.1. Data analysis was carried out using SPSS version 20.0 and STATA version 15.0. p-value $\leq 0.05$ was reported as statistically significant. Spatial distribution analysis was done using ArcGIS system for Geographical Information System (GIS).

Result: The overall prevalence of S. mansoni infection was $36.6 \%$ (Cl: $32-41.9)$ with intensity of $30.5 \%$, $27.0 \%$ and $42.6 \%$ for light, moderate, and heavy, respectively. Among the different fresh water snails collected on the basis of shell morphology 546(\%) were Biomphalaria pfeifferi, 310(28.1\%) were Bulinus spp, 101(9.1\%) were Lymnaea, and 147(13.3\%) were Bivalve. Schistosome infection in Biomphalaria spp. was $0.0 \%$. Swimming frequency and proximity to water body were observed to be the most associated risk factor to $S$. mansoni infection.

Conclusion: The study showed that S. mansoni is an ongoing health problem in Gorgora. Different fresh water snails with a potential of transmitting diseases of human and veterinary importance that requires attention had been identified. Longitudinal and molecular study on Biomphalaria pfeifferi infection rate as well as susceptibility to schistosome infection is recommended.

\section{Background}

Schistosomiasis is one of the great neglected and unconquered tropical diseases caused by the digenetic trematode Schistosoma (1). It is an important socio economic and public health problem worldwide that is mostly prevalent in many developing countries where poor sanitation and low access to safe drinking water is predominant (2). Schistosoma haematobium, Schistosoma japonicum, and Schistosoma mansoni are the three main species infecting humans and $S$. mekongi and $S$. intercalatum are restricted in certain geographical locations. Schistosoma mansoni and S. haematobium which causes intestinal and urinary schistosomiasis respectively, are the causes of African schistosomiasis(2,3). Transmission 
of schistosomiasis involves the release of ova from infected hos faces or urine depending on the species, hatching of ova that release miracidia which ends up in the infection of appropriate intermediate snail host. Human infection involves skin penetration by cercaria when coming in contact with water containing Schistosome-infected snails. Inside the human host, cercaria develop into sexually mature ova-laying worms in the hepatic portal system (4).

Freshwater snails play salient role in the ecology of fresh-water; serving as food for numerous other animals and feeds on vast amounts of algae and detritus. Whereas estimated 350 snail specie play significant role in public and veterinary health, many species of freshwater snail belonging to the family Planorbidae serve as intermediate hosts for a number of infections such as angiostrongyliasis, clonorchiasis, fascioliasis, fasciolopsiasis, opisthorchiasis, paragonimiasis and the most common, schistosomiasis in Africa, Asia and the Americas $(5,6)$. Human Schistosoma parasites species are transmitted by a responsible intermediate hosts: Biomphalaria, Bulinus, and Oncomelania for S. mansoni, S. haematobium, and S. japonicum, respectively $(7,8)$.

Schistosomiasis mostly affects poor and rural communities, particularly agricultural and fishing populations. Women doing domestic chores in infested water, such as washing clothes, are also at risk and can develop female genital schistosomiasis. Inadequate hygiene and contact with infected water make children especially vulnerable to infection(11).

Mass drug administration with Praziquantel (PZQ) is the cornerstone in schistosomiasis control strategies in which extensive reliance on just one drug is scaling up and this leads to emergence of resistance due to selective pressure $(12,13)$. Recent reports on the possible development of resistance and treatment failure to PZQ have generated much more unrest.

In Ethiopia, while S. mansoni is wide spread, S. hematobium is restricted to few areas. Although there are ample literatures on the distribution and infection status of schistosomiasis in Ethiopia, there is a need to conduct study on the distribution and infection status of snail intermediate hosts (3).Therefore, the availability of this information is important in designing effective disease control measures such as mollucicidal chemicals.

\section{Methods}

A Community based cross-sectional study was conducted from May 2020 to March 2020 in selected Schistosome endemic village in Gorgora Town of Western Dembia District Northwest, Ethiopia. Gorgora is a small road side town on the shore of Lake Tana, $818 \mathrm{Km}$ away from Addis Ababa. The average temperature ranges from $29{ }^{\circ} \mathrm{C}$ to $31^{\circ} \mathrm{C}$. Annual rainfall ranges from $900 \mathrm{~mm}$ to $1038 \mathrm{~mm}$, with Altitude of $1800 \mathrm{~m}$ to $1900 \mathrm{~m}$ above sea level and located at $10^{\circ} 17^{\prime} \mathrm{N}$ latitude and $37^{\circ} 26^{\prime} \mathrm{E}$ longitude. The population is estimated to be 2,500 with $2.89 \mathrm{~km}$ square area (16)

The parasitological examination sample size was determined by using the single population proportion formula with the following assumptions: prevalence $(p)$ of $50 \%$ to increase sample size, $95 \%$ confidence 
level, $5 \%$ margin of error, and a sample estimation correction formula was considered. Moreover, a 10\% non-response rate was added and the final sample size $(n)$ was $=385$.

A multi-stage sampling procedure was utilized to select study participants. At the first stage, village was selected randomly by simple random sampling from three villages. At the second sampling stage, numbers of households included in each village was determined by proportional allocation according to the total number of households found in each village/kebeles. Then, systematic sampling method was employed to select the households. The sampling interval was calculated by dividing the total households to the number of households to be included in the sample for each village. The initial household was randomly selected by lottery method and the next households was selected at that interval. Whenever more than one eligible was found in the same selected household, only one of them was chosen using the lottery method. In case no eligible candidate was identified in a selected household, the next household was selected keeping the interval constant afterwards. In addition, a person in the selected households was enrolled based on inclusion criteria. Then each data collector conducted a house-to house survey. Each water point and study households was Geo-referenced using Garmin64.

\section{Data collection and laboratory methods}

\section{Malacological survey}

All sites which showed signs of human activity near the shoreline were selected for snail collection. Intermediate snail hosts was surveyed both in the lake and in the stream at human water contact sites. In the likely habitats, observations was also made on physical characteristics such as vegetation abundance, turbidity, the nature of the substrate and stones. In addition, the meantime water contact activities were also surveyed(4).Snails were collected by scooping, hand picking using forceps and gloves and put in open plastic bucket that contained a small quantity of water and weeds. Thereafter, snails were transported to Medical Parasitology laboratory, College of Medicine and Health Sciences, University of Gondar for determination of infection and identification. Then, snails were examined for natural trematode infections by shedding method(18). Each snail was placed individually in the shedding vials with water (deionized water) and then exposed to electric light for about one hour. The cercariae shed by the snails was identified to the genus level by their tail morphology following WHO recommendation $(5,6)$.

\section{Socio demography and risk factor assessment}

Well-structured questionnaire based on the known risk factors for Schistosoma was prepared in Amharic and was translated later in English version. It was translated later into the Amharic, local language of the study area. The questionnaire addresses inhabitant's socio-demographic information and risk factors. A pretest was conducted and additional response categories was added based on the pretest findings.

\section{Parasitological Techniques}

A single stool specimen of about $2 \mathrm{~g}$ was collected from each study participant using clean, dry and leak proof plastic container labeled with unique identification number. A portion of the sample was processed 
by Kato-Katz method using a template holding $41.7 \mathrm{mg}$ of stool(19). Kato-Katz smears were processed in study sites and slides were transported and examined under a microscope at University of Gondar (UoG), Medical Parasitology Laboratory. A small portion of the stool samples was preserved in $10 \%$ formalin for repeating for formol-ether concentration techniques $(19,20)$. Examination for hookworm was performed within one hour of stool collection and Kato-Katz slide preparation. The slides were left for $24 \mathrm{~h}$ to clear for easy visualization of $S$. mansoni and other helminthes eggs. After $24 \mathrm{~h}$, two experienced laboratory technologists examined each slides independently. Whenever the result of the two laboratory technologists discorded, the third experienced laboratory technologist examined the slides. Results of the laboratory investigation was recorded on a format prepared for this purpose. Infection intensity of the $S$. mansoni and STHs was estimated by multiplying the total number of eggs counted by 24 , which gives as the eggs per gram (epg) of stool. Besides, the species specific classes of infection intensity with $S$. mansoni and STH were classified as light, moderate and heavy per the threshold set by WHO (21). Inhabitants who were found positive for intestinal parasites infection were treated with standard dose of anti helmintic treatment(22).

\section{Evaluation of physicochemical parameters}

The evaluation of the physico-chemical parameters of water bodies was carried out to a depth not exceeding 0.5 meters. Using conductivity $(\mu \mathrm{S} / \mathrm{cm})$ multi-parameter digital probe recorder (HANNA instruments). Then the tube was placed in the device for 3 minutes to obtain the value. Hydric potential $(\mathrm{pH})$ was obtained using $\mathrm{pH}$-meter (HANNA instruments) by immersion of a probe. The water temperature $\left({ }^{\circ} \mathrm{C}\right)$ was measured by a flowatch (HANNA instruments) equipped with a submerged propeller at a depth of 0.5 meters $(7)$.

\section{Data Analysis and Interpretation}

Data were double entered, coded, cleaned, and verified using EpiData Version 4.4.2.1 software (EpiData Association, Odense, Denmark). Data analysis was carried out using SPSS version 20.0 (IBM Corp. IBM SPSS Statistics for Windows, Armonk, NY, USA) and STATA version 15.0 (StataCorp. Statistical Software: College Station, TX 77845, USA). Different variables were described and characterized by frequency distribution $(n(\%))$. Binary and Multivariable logistic regression analysis was done for associated risk factors for acquiring S. mansoni infection. Distribution analysis was done using ArcGIS system for Geographical Information System (GIS) for proximity analysis. The prevalence of schistosomiasis was computed as percentage (8).

\section{Results}

\section{Socio-demographic characteristics}

A total of 385 study participants of which 189 (49.1\%) males and 196 (50.9\%) females were participated in the study (Table 1). All the study participants were urban residents in Gorgora town. The mean age of 
participants was (23.84) years. Age of the participants ranged from 1 to 80 years (SD \pm 17.547 ) 
Table 1

Socio-demographic characteristics of study participants at Gorgora town, Western Dembia, Northwest Ethiopia 2020.

\begin{tabular}{|c|c|c|c|}
\hline \multicolumn{2}{|l|}{ Socio-demographic variables } & \multirow{2}{*}{$\begin{array}{l}\text { Frequency } \\
189\end{array}$} & \multirow{2}{*}{$\begin{array}{l}\text { Percentage } \\
49.1 \%\end{array}$} \\
\hline Sex & Male & & \\
\hline & Female & 196 & $50.9 \%$ \\
\hline \multirow[t]{9}{*}{ Age } & $<4$ & 35 & $9.1 \%$ \\
\hline & $5-9$ & 52 & $13.5 \%$ \\
\hline & $10-14$ & 76 & $19.7 \%$ \\
\hline & $15-19$ & 21 & $5.5 \%$ \\
\hline & $20-24$ & 30 & $7.8 \%$ \\
\hline & $25-29$ & 56 & $14.5 \%$ \\
\hline & $30-34$ & 26 & $6.6 \%$ \\
\hline & $35-39$ & 18 & $4.8 \%$ \\
\hline & $>40$ & 71 & $18.4 \%$ \\
\hline \multirow[t]{4}{*}{ Educational level } & Illiterate & 106 & $27.5 \%$ \\
\hline & Primary & 167 & $43.4 \%$ \\
\hline & Secondary & 85 & $22.1 \%$ \\
\hline & Tertiary & 27 & $7.0 \%$ \\
\hline \multirow[t]{4}{*}{ Occupational status } & Not employed & 266 & $69.1 \%$ \\
\hline & Self employed & 88 & $22.9 \%$ \\
\hline & Government employed & 26 & $6.8 \%$ \\
\hline & Private employed & 5 & $1.3 \%$ \\
\hline \multirow[t]{4}{*}{ Average monthly income (Birr) } & $<1000$ & 309 & 80. \\
\hline & $1001-2000$ & 53 & $13.8 \%$ \\
\hline & $2001-3000$ & 12 & $3.1 \%$ \\
\hline & $>3000$ & 11 & $2.9 \%$ \\
\hline \multirow[t]{3}{*}{ Marital status } & Married & 140 & $36.4 \%$ \\
\hline & Single & 226 & $58.7 \%$ \\
\hline & Divorced & 11 & $2.9 \%$ \\
\hline
\end{tabular}




\section{Prevalence of $S$. mansoni infections by demographic characteristics}

Schistosoma mansoni infection was observed in 141(36.6\%) study participants. Of the 141 S. mansoni infected $76(40.2 \%)$ were males and $65(33.3 \%)$ were females. Out of 385 total participants examined, 208(54.0\%) were positive for at least single helminth infection; and among the seven helminths identified, the prevalence of other helminthic parasites were $17.4 \%$ of which Ascaris lumbricoides $(14.1 \%)$ was the highest followed by hookworm (2\%). One hundred ninthly four (50.4\%) study participants had single infection and $14(3.6 \%)$ had multiple infections (Table 2).

Table 2

Sex distribution of $S$. mansoni and intestinal helminths infections among study subjects of Gorgora western Dembia, Northwest Ethiopia 2020.

\begin{tabular}{|llll|}
\hline Types Helminths Parasites & \multicolumn{3}{l}{ Kato-Katz technique result $(\mathbf{n}=\mathbf{3 8 5})$} \\
\cline { 2 - 4 } & $\begin{array}{l}\text { Male N\% } \\
(\mathbf{n}=\mathbf{1 8 9})\end{array}$ & $\begin{array}{l}\text { Female N\% } \\
(\mathbf{n}=196)\end{array}$ & $\begin{array}{l}\text { Total N\% } \\
(\mathbf{n}=385)\end{array}$ \\
\hline Schistosoma mansoni & $76(40.2)$ & $65(33.2)$ & $141(36.6)$ \\
\hline Ascaris lumbricoides & $27(14.3)$ & $27(13.8)$ & $54(14.1)$ \\
\hline Hookworm & $4(2.1)$ & $4(2.0)$ & $8(2.1)$ \\
\hline At least one parasite infection & $111(58.7)$ & $97(49.5)$ & $208(54.0)$ \\
\hline Single infection & $104(55.0)$ & $90(45.9)$ & $194(50.4)$ \\
\hline Multiple infection & $7(3.7)$ & $7(3.6)$ & $14(3.6)$ \\
\hline
\end{tabular}

\section{Assessing risk factors for $S$. mansoni infection}

Among 385 participants 58(15.1\%), 207(53.8\%), and 120(31.2\%) had reported none, sometimes and frequent contact with Lake Tana, respectively. Of this $16(11.3 \%), 67(45.5 \%)$ and $58(41.1 \%)$ were infected with $S$. mansoni. In bivariate analysis, risk factors associated with ( $p$-value $>0.05$ ) and those variables with ( $p$-value $<0.25$ ) were carried to multiple logistic regression to see independent effect of variables. The odds of getting $S$. mansoni infection among individuals swimming sometimes was 7.7 times higher than those they didn't have an exposure of swimming at Lake Tana (AOR: 7.7; 95\% Cl: 2.42-24.5; $p=$ 
0.001). The odds of getting $S$. mansoni infection among individuals swimming frequently was 10 times higher than those they didn't have an exposure of swimming at Lake Tana (AOR: 10.0; 95\% Cl: 3.19-36.6; $p=0.000$ ). The odds of getting $S$. mansoni infection among individuals living $1 \mathrm{~km}$ away from the shoreline was 1.7 times higher than those who are living $1 \mathrm{~km}$ away from the shoreline at Lake Tana (AOR: $1.70,95 \% \mathrm{Cl}: 1.03-2.84 ; \mathrm{p}=0.039$ ). The odds of getting $S$. mansoni infection among individuals Fishing and Farming was 7.9 times higher than those they didn't have an exposure of fishing and farming at Lake Tana (AOR: 7.9, 95\%Cl: 2.15-29.1; $=0.002$ ) (Table 3). 
Table 3

Binary and Multivariable logistic regression analysis of factors associated to $S$. mansoni infection in Gorgora town, Western, Dembia District, Northwest Ethiopia 2020.

\begin{tabular}{|c|c|c|c|c|c|c|}
\hline \multirow[t]{2}{*}{ Variables } & \multicolumn{2}{|c|}{ Schistosome infection } & \multirow{2}{*}{$\begin{array}{l}\text { Frequency } \\
\text { No. (\%) } \\
(n=385)\end{array}$} & \multicolumn{2}{|l|}{ OR $(95 \% \mathrm{Cl})$} & \multirow[t]{2}{*}{ P-value } \\
\hline & $\begin{array}{l}\text { Positive } \\
\text { N (\%) }\end{array}$ & $\begin{array}{l}\text { Negative } \\
\mathbf{N}(\%)\end{array}$ & & COR & AOR & \\
\hline \multicolumn{7}{|l|}{ Sex } \\
\hline Female & $65(33.3)$ & $131(66.8)$ & 196(50.9) & $1^{*}$ & & \\
\hline Male & $76(40.2)$ & 113(59.8) & $189(49.1)$ & $\begin{array}{l}1.35(0.89- \\
2.05)\end{array}$ & $\begin{array}{l}1.19(0.7- \\
2.04)\end{array}$ & 0.532 \\
\hline \multicolumn{7}{|l|}{ Age } \\
\hline $1-4$ & $26(10.7)$ & $9(6.4)$ & $35(9.1)$ & $1^{\star}$ & & \\
\hline $5-9$ & 41(16.8) & $11(7.8)$ & $52(13.5)$ & $\begin{array}{l}0.77(0.28- \\
2.12)\end{array}$ & $\begin{array}{l}0.51(0.15- \\
1.76)\end{array}$ & 0.289 \\
\hline $10-14$ & $39(16.0)$ & $37(26.2)$ & 76(19.7) & $\begin{array}{l}2.74(1.14- \\
6.62)\end{array}$ & $\begin{array}{l}1.4(0.4- \\
4.61)\end{array}$ & 0.629 \\
\hline $15-19$ & $12(4.9)$ & $9(6.4)$ & $21(5.5)$ & $\begin{array}{l}2.26(0.68- \\
6.83)\end{array}$ & $\begin{array}{l}1.05(0.39- \\
4.69)\end{array}$ & 0.940 \\
\hline $20-24$ & $18(7.4)$ & $12(8.5)$ & $30(7.8)$ & $\begin{array}{l}1.93(0.67- \\
5.52)\end{array}$ & $\begin{array}{l}0.70(0.17- \\
2.83)\end{array}$ & 0.624 \\
\hline $25-29$ & $36(14.8)$ & $20(14.2)$ & $56(14.5)$ & $\begin{array}{l}1.60(0.63- \\
4.08)\end{array}$ & $\begin{array}{l}0.49(0.13- \\
1.83)\end{array}$ & 0.294 \\
\hline $30-34$ & $11(4.5)$ & $15(10.6)$ & $26(6.8)$ & $\begin{array}{l}3.94(1.33- \\
11.67)\end{array}$ & $\begin{array}{l}1.09(0.3- \\
4.46)\end{array}$ & 0.894 \\
\hline $35-39$ & $9(3.7)$ & $9(6.4)$ & $18(4.7)$ & $\begin{array}{l}2.88(0.87- \\
9.54)\end{array}$ & $\begin{array}{l}0.94(0.20- \\
4.35)\end{array}$ & 0.940 \\
\hline$>40$ & $52(21.3)$ & 19(13.5) & 71(18.4) & $\begin{array}{l}1.05(0.41- \\
2.65)\end{array}$ & $\begin{array}{l}0.56(0.10- \\
1.75)\end{array}$ & 0.323 \\
\hline \multicolumn{7}{|c|}{ Educational level } \\
\hline Illiterate & $29(27.4)$ & 77(72.6) & $106(27.5)$ & $1^{*}$ & & \\
\hline Primary & 64(38.3) & 103(61.7) & $167(43.4)$ & $\begin{array}{l}1.64(0.97- \\
2.79)\end{array}$ & $\begin{array}{l}0.95(0.44- \\
2.01)\end{array}$ & 0.896 \\
\hline Secondary & $36(42.4)$ & $49(57.6)$ & $85(22.1)$ & $\begin{array}{l}1.95(1.06- \\
3.57)\end{array}$ & $\begin{array}{l}1.18(0.50- \\
2.79)\end{array}$ & 0.694 \\
\hline
\end{tabular}

(Note: COR: crude odd ratio; Cl: confidence interval; AOR: adjusted odd ratio; * Reference category, **Significant association.) 


\begin{tabular}{|c|c|c|c|c|c|c|}
\hline \multirow[t]{2}{*}{ Variables } & \multicolumn{2}{|c|}{ Schistosome infection } & \multirow{2}{*}{$\begin{array}{l}\text { Frequency } \\
\text { No. }(\%) \\
(n=385)\end{array}$} & \multicolumn{2}{|l|}{ OR $(95 \% \mathrm{Cl})$} & \multirow[t]{2}{*}{ P-value } \\
\hline & $\begin{array}{l}\text { Positive } \\
\text { N (\%) }\end{array}$ & $\begin{array}{l}\text { Negative } \\
\mathbf{N}(\%)\end{array}$ & & COR & AOR & \\
\hline Tertiary & $12(44.4)$ & $15(55.6)$ & $27(7.0)$ & $\begin{array}{l}2.12(0.88- \\
5.07)\end{array}$ & $\begin{array}{l}2.7(0.68- \\
10.7)\end{array}$ & 0.156 \\
\hline \multicolumn{7}{|l|}{ Occupational status } \\
\hline Not employed & $88(33.1)$ & 178(66.9) & $266(69.1)$ & $1 *$ & & \\
\hline Self employed & $41(46.6)$ & $47(53.4)$ & $88(22.9)$ & $\begin{array}{l}1.76(1.08- \\
2.88)\end{array}$ & $\begin{array}{l}1.7(0.79- \\
3.09)\end{array}$ & 0.164 \\
\hline Govt' employed & $10(38.5)$ & $16(61.5)$ & $26(6.8)$ & $\begin{array}{l}1.26(0.55- \\
2.90)\end{array}$ & $\begin{array}{l}1.4(0.29- \\
7.33)\end{array}$ & 0.645 \\
\hline Private employed & $2(40.0)$ & $3(60.0)$ & $5(1.3)$ & $\begin{array}{l}1.34(0.22- \\
8.22)\end{array}$ & $\begin{array}{l}1.9(0.23- \\
16.18)\end{array}$ & 0.543 \\
\hline \multicolumn{7}{|c|}{ Average monthly income } \\
\hline$<1000$ & 106(34.3) & $203(65.7)$ & $309(80.3)$ & $1 *$ & & \\
\hline $1001-2000$ & $29(54.7)$ & $24(45.3)$ & $53(13.8)$ & $\begin{array}{l}2.31(1.28- \\
4.17)\end{array}$ & $\begin{array}{l}1.25(0.48- \\
3.26)\end{array}$ & 0.641 \\
\hline $2001-3000$ & $4(33.3)$ & $8(66.7)$ & $12(3.1)$ & $\begin{array}{l}0.95(0.28- \\
3.25)\end{array}$ & $\begin{array}{l}0.58(0.09- \\
3.89)\end{array}$ & 0.578 \\
\hline$>3000$ & $2(18.2)$ & $9(81.8)$ & $11(2.9)$ & $\begin{array}{l}0.43(0.09- \\
2.01)\end{array}$ & $\begin{array}{l}0.22(0.19- \\
2.47)\end{array}$ & 0.221 \\
\hline \multicolumn{7}{|c|}{ Household drinking water source } \\
\hline Lake & $1(16.7)$ & $5(83.3)$ & $6(1.6)$ & $1^{*}$ & & \\
\hline Spring & $3(50.0)$ & $3(50.0)$ & $6(1.6)$ & $\begin{array}{l}4.99(0.34- \\
72.7)\end{array}$ & $\begin{array}{l}2.9(0.14- \\
59.7)\end{array}$ & 0.486 \\
\hline Pipe Water & 137(36.7) & 236(63.3) & $373(96.9)$ & $\begin{array}{l}2.90(0.33- \\
25.1)\end{array}$ & $\begin{array}{l}2.71(0.24- \\
29.6)\end{array}$ & 0.415 \\
\hline \multicolumn{7}{|l|}{ Lake } \\
\hline Washing & $117(36.3)$ & $205(63.7)$ & $322(83.6)$ & $1^{*}$ & & \\
\hline Collecting water & $6(37.5)$ & $10(62.5)$ & $16(4.2)$ & $\begin{array}{l}1.01(0.36- \\
2.81)\end{array}$ & $\begin{array}{l}1.2(0.40- \\
3.98)\end{array}$ & 0.605 \\
\hline Swimming/Playing & $6(37.5)$ & $10(62.5)$ & $16(4.2)$ & $\begin{array}{l}0.76(0.40- \\
1.43)\end{array}$ & $\begin{array}{l}0.68(0.33- \\
1.38)\end{array}$ & 0.290 \\
\hline
\end{tabular}

(Note: COR: crude odd ratio; Cl: confidence interval; AOR: adjusted odd ratio; * Reference category, **Significant association.) 


\begin{tabular}{|c|c|c|c|c|c|c|}
\hline \multirow[t]{2}{*}{ Variables } & \multicolumn{2}{|c|}{ Schistosome infection } & \multirow{2}{*}{$\begin{array}{l}\text { Frequency } \\
\text { No. (\%) } \\
(n=385)\end{array}$} & \multicolumn{2}{|l|}{ OR (95\% Cl) } & \multirow[t]{2}{*}{ P-value } \\
\hline & $\begin{array}{l}\text { Positive } \\
\text { N (\%) }\end{array}$ & $\begin{array}{l}\text { Negative } \\
\mathbf{N}(\%)\end{array}$ & & COR & AOR & \\
\hline Fishing/Farming & 12(38.7) & 19(61.3) & $31(8.1)$ & $\begin{array}{l}9.9(2.81- \\
37.7)^{\star \star}\end{array}$ & $\begin{array}{l}7.9(2.15- \\
29.1)^{\star \star}\end{array}$ & $0.002^{* *}$ \\
\hline \multicolumn{7}{|l|}{ Latrine practices } \\
\hline No & $140(36.6)$ & $240(63.4)$ & $382(99.2)$ & $1 *$ & & \\
\hline Yes & $1(33.3)$ & $2(66.7)$ & $3(0.8)$ & $\begin{array}{l}0.86(0.08- \\
9.61)\end{array}$ & $\begin{array}{l}1.6(0.07- \\
52.6)\end{array}$ & 0.801 \\
\hline \multicolumn{7}{|c|}{ Swimming frequency } \\
\hline None & $4(2.8)$ & $54(22.1)$ & $58(15.1)$ & $1 *$ & & \\
\hline Sometimes & $79(56.0)$ & $128(52.5)$ & 207(53.8) & $\begin{array}{l}8.3(2.91- \\
23.9)^{\star \star}\end{array}$ & $\begin{array}{l}7.7(2.42- \\
24.5)^{\star \star}\end{array}$ & $0.001^{* *}$ \\
\hline Frequent & $58(41.1)$ & $62(25.4)$ & $120(31.2)$ & $\begin{array}{l}12.6(4.30- \\
37.1)^{\star \star}\end{array}$ & $\begin{array}{l}10.0(3.19- \\
36.6)^{\star \star}\end{array}$ & $0.000^{* *}$ \\
\hline \multicolumn{7}{|c|}{ Proximity to Lake Tana } \\
\hline$<1 \mathrm{~km}$ & 115(81.6) & $176(72.1)$ & 291(75.6) & $\begin{array}{l}1.70(1.03- \\
2.84)^{\star \star}\end{array}$ & $\begin{array}{l}1.89(1.05- \\
3.36)^{\star \star}\end{array}$ & $0.033^{\star *}$ \\
\hline$\geq 1-1.5 \mathrm{~km}$ & $26(18.4)$ & $68(27.9)$ & $94(24.4)$ & $1 *$ & & \\
\hline
\end{tabular}

\section{Intensity of $S$. mansoni infection}

Among S. mansoni infected study participant (141/385) 30.5\%, $27.0 \%$ and $42.6 \%$ had light, moderate, and heavy intensity of infection, respectively. Of the total 60 (42.6\%) heavy infections of $S$. mansoni, the highest $7(11.7 \%), 7(11.7 \%)$, was observed in participants of the aged group of $10-14$ and, $>40$ years, respectively followed by $6(10.0 \%)$ and $6(10.0 \%)$ in adolescent $25-29$ and 30-34years, respectively. The heavy infections to $S$. mansoni in males and females were $19(50.0 \%)$ and $19(50.0 \%)$, respectively. The highest egg count was 3600 egg per gram of stool (Table 4). 
Table 4

Intensity of infections for S. mansoni among participants in Gorgora

town, Western Dembia, Northwest Ethiopia 2020.

\begin{tabular}{|c|c|c|c|c|c|}
\hline \multicolumn{2}{|c|}{ Variables } & \multirow{2}{*}{\multicolumn{3}{|c|}{$\begin{array}{l}\text { Parasites identified (S. manson }{ }^{*} \text { ) } \\
\text { Classes of intensity }(n=141)\end{array}$}} & \multirow{4}{*}{$\begin{array}{l}\text { Total } \\
\mathrm{N}(\%)\end{array}$} \\
\hline & & & & & \\
\hline & & \multirow{2}{*}{$\begin{array}{l}\text { Light } \\
N(\%)\end{array}$} & \multirow{2}{*}{$\begin{array}{l}\text { Moderate } \\
N(\%)\end{array}$} & \multirow{2}{*}{$\begin{array}{l}\text { Heavy } \\
N(\%)\end{array}$} & \\
\hline & & & & & \\
\hline \multirow[t]{9}{*}{ Age } & $1-4$ & $3(7.0)$ & $4(10.5)$ & 2(3.3) & $9(6.4)$ \\
\hline & $5-9$ & $4(9.3)$ & $3(7.9)$ & $4(6.7)$ & $11(7.8)$ \\
\hline & $10-14$ & 10(23.3) & $20(52.6)$ & 7(11.7) & $37(26.2)$ \\
\hline & $15-19$ & $2(4.7)$ & $5(13.2)$ & 2(3.3) & $9(6.4)$ \\
\hline & $20-24$ & $6(14.0)$ & $5(13.2)$ & $1(1.7)$ & $12(8.5)$ \\
\hline & $25-29$ & $5(11.6)$ & $9(20.9)$ & $6(10.0)$ & $20(14.2)$ \\
\hline & $30-34$ & $5(11.6)$ & $4(10.5)$ & $6(10.0)$ & 15(10.6) \\
\hline & $35-39$ & $2(4.7)$ & $4(10.5)$ & $3(5.0)$ & $9(6.4)$ \\
\hline & $>40$ & $6(14.0)$ & $6(15.8)$ & $7(11.7)$ & $19(13.5)$ \\
\hline \multirow[t]{2}{*}{ Sex } & Male & $23(53.5)$ & $34(56.7)$ & 19(50.0) & 76(53.9) \\
\hline & Female & $20(46.5)$ & $26(43.3)$ & 19(50.0) & $65(46.1)$ \\
\hline \multicolumn{2}{|c|}{ Total } & $43(30.5)$ & $38(27.0)$ & $60(42.6)$ & $141(100)$ \\
\hline \multicolumn{6}{|c|}{ * Light (1-99 epg), Moderate (100-399 epg), Heavy ( $\geq 400 \mathrm{epg})$. } \\
\hline
\end{tabular}

\section{Spatial distribution of $S$. mansoni and Malacological surveys}

A total of 385 study participant (141 positives for S. mansoni) participated in the study, and their household and snail collection area geo-location was mapped (Fig. 3). Spatial distribution of intermediate snail hosts of human schistosomes in the Lake Tana provided insights on the diversity of snail and their density. S. mansoni infections were clustered near the eastern edge of the surveillance area. 
Among 385 study participant who had 120(31.2\%), 207(53.3\%) and 58(15.1\%) had frequently sometimes and none swimming frequency respectively. Of 141 positives for $S$. mansoni participants of this $58(41.1 \%), 79(56.0 \%)$ and $4(2.8 \%)$ had frequently, sometimes and none swimming frequency respectively, (Table 5).

\section{Table 5}

Prevalence of $S$. mansoni among study participants self-reported swimming frequency per week with the shoreline of Lake Tana, in Gorgora town, Western Dembia, Northwest Ethiopia 2020.

\begin{tabular}{|c|c|c|c|c|}
\hline \multirow{3}{*}{$\begin{array}{l}\text { Parasite } \\
\text { finding }\end{array}$} & \multicolumn{3}{|c|}{ Swimming frequency } & \multirow{3}{*}{$\begin{array}{l}\text { Total } \\
\text { N\% }\end{array}$} \\
\hline & $\begin{array}{l}\text { None (0 per } \\
\text { week) }\end{array}$ & $\begin{array}{l}\text { Sometimes ( } 1 \text { per } \\
\text { week) }\end{array}$ & $\begin{array}{l}\text { Frequent ( } 2-3 \text { per } \\
\text { week) }\end{array}$ & \\
\hline & N\% & N\% & N\% & \\
\hline Positive & $4(2.8 \%)$ & $79(56.0 \%)$ & $58(41.1 \%)$ & $141(36.6 \%)$ \\
\hline Negative & $54(21.1 \%)$ & $128(52.5 \%)$ & $62(25.4)$ & $244(63.4 \%)$ \\
\hline Total & $58(15.1 \%)$ & $207(53.3 \%)$ & $120(31.2 \%)$ & $385(100)$ \\
\hline
\end{tabular}

\section{Human infection rate in proximity to water point}

Out of $94(24.4 \%)$ who are living beyond $1-1.5 \mathrm{~km}$ from the shoreline of Lake Tana 15(10.6\%) positive for S. mansoni of this $6(40.0 \%), 8(53.3 \%)$ and $1(6.7 \%)$ had frequently, sometimes and none swimming frequency respectively.

Table 6

Prevalence of $S$. mansoni among study participants swimming frequency and proximity to the shoreline of Lake Tana, in Gorgora town, Western Dembia, Northwest Ethiopia 2020.

\begin{tabular}{|c|c|c|c|c|c|c|}
\hline \multirow{2}{*}{$\begin{array}{l}\text { Lake Tana } \\
\text { Contact }\end{array}$} & \multicolumn{2}{|l|}{$<1 \mathrm{~km}$} & \multicolumn{3}{|c|}{$\geq 1-1.5 \mathrm{~km}$} & \multirow{2}{*}{$\begin{array}{l}\text { Total } \\
\begin{array}{l}\text { No. } \\
\text { Tested }\end{array}\end{array}$} \\
\hline & $\begin{array}{l}\text { No. } \\
\text { Tested }\end{array}$ & $\begin{array}{l}\text { No..Positive } \\
\text { (\%) }\end{array}$ & $\begin{array}{l}\text { No. } \\
\text { Tested }\end{array}$ & $\begin{array}{l}\text { No..Positive } \\
\text { (\%) }\end{array}$ & $\begin{array}{l}\text { No..Positive } \\
\text { (\%) }\end{array}$ & \\
\hline None & $50(17.2)$ & $3(2.4)$ & $8(8.5)$ & $1(6.7)$ & $4(3.0)$ & $58(15.1)$ \\
\hline Some & 157(54.0) & $71(56.3)$ & $50(53.2)$ & $8(53.3)$ & $79(56.0)$ & $207(53.8)$ \\
\hline Frequent & $84(28.9)$ & $52(41.3)$ & $36(38.3)$ & $6(40.0)$ & $58(41.1)$ & $120(31.2)$ \\
\hline Total & $291(75.6)$ & $126(89.4)$ & $94(24.4)$ & $15(10.6)$ & $141(36.6)$ & $385(100)$ \\
\hline
\end{tabular}

\section{S. mansoni intensity of infections in proximity to water point}


Out of 385/Two hundred ninety-one $75.6 \%$ participants lived in less than $1 \mathrm{~km}$ from the shoreline Lake Tana. From 291 participants lived in less than $1 \mathrm{~km} 81.6 \%$ of $S$. mansoni positive cases are found in this area while $18.4 \%$ of the infections occurred beyond $1 \mathrm{~km}$ distance (Table 7 ).

Table 7

Intensity of S. mansoni infection and proximity of study participant from the shoreline of Lake Tana, in Gorgora town, Western Dembia, Northwest Ethiopia 2020

\begin{tabular}{|llllll|}
\hline \multirow{2}{*}{ Distance (km) } & Tested No. (\%) & \multicolumn{3}{l}{ Intensity of infections No. (\%) } & Total infected \\
\cline { 3 - 5 } & & Light & Moderate & Heavy & No. (\%) \\
& & $\mathbf{N} \%$ & $\mathbf{N} \%$ & $\mathbf{N} \%$ & \\
\hline$<1 \mathrm{~km}$ & $291(75.6)$ & $33(28.7)$ & $49(42.6)$ & $33(28.7)$ & $115(81.6)$ \\
\hline$\geq 1-1.5 \mathrm{~km}$ & $94(24.4)$ & $10(38.5)$ & $11(42.3)$ & $5(19.2)$ & $26(18.4)$ \\
\hline Total & $385(100)$ & 43(30.5) & $60(42.6)$ & $38(27.0)$ & $141(36.7)$ \\
\hline * Light 1-99 eggs per gram (epg); Moderate & 100-399 epg; Heavy $\geq 400$ epg. \\
\hline
\end{tabular}

\section{Snail species, distribution, abundance and Infection Rates}

A total of 1,105 freshwater snail specimens were collected from 14 different sampling sites along the shores of Lake Tana. Out of this 546 were Biomphalaria pfeifferi. Among the living 500 Biomphalaria snails 492 were not observed to shed human schistosome cercaria, while $8(1.6 \%)$ shed bird's schistosome cercariae. Although, the highest snail abundance was observed in Fish Farm and Military Camp (FF \& MC), where 220 snails were collected representing $40.2 \%$ of the total Biomphalaria spp. collected in the village, this habitat had neither the highest number nor the highest prevalence of snails with patent infection, with only $8(1.6 \%)$ shedding bird schistosome cercariae (Table 8 ). 
Table 8

Total number of snail population collected and infestation rate in Lake Tana, in Gorgora town, Western Dembia, Northwest Ethiopia 2020.

\begin{tabular}{|c|c|c|c|c|c|}
\hline $\begin{array}{l}\text { Habitat } \\
\text { no. }\end{array}$ & Habitat name & $\begin{array}{l}\text { Total No. of } \\
\text { Snail collected }\end{array}$ & $\begin{array}{l}\text { No. of snails } \\
\text { Biomphalaria } \\
\text { (\%) }\end{array}$ & $\begin{array}{l}\text { Tested Spp. } \\
\text { Biomphalaria }\end{array}$ & $\begin{array}{l}\text { No. of } \\
\text { snails } \\
\text { infested } \\
\text { rate (\%) }\end{array}$ \\
\hline 1 & $\begin{array}{l}\text { Deber Sina (DS1 \& } \\
\text { DS2)* }\end{array}$ & $65(5.9)$ & $61(11.2)$ & $60(12.0)$ & $1(1.7)$ \\
\hline 2 & 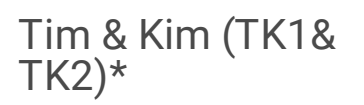 & $222(20.1)$ & $67(12.3)$ & $60(12.0)$ & $2(3.3)$ \\
\hline 3 & $\begin{array}{l}\text { Military Camp 5th } \\
\text { (MC1\& MC2)* }^{*}\end{array}$ & $298(26.9)$ & $116(21.2)$ & $100(20.0)$ & $3(3)$ \\
\hline 4 & $\begin{array}{l}\text { Fish Farm Center } \\
(\text { FF1 \& FF2)* }\end{array}$ & $297(26.9)$ & 104(19.0) & $100(20.0)$ & $2(2)$ \\
\hline 5 & $\begin{array}{l}\text { Port Hotel (PH1\& } \\
\text { PH2)* }\end{array}$ & $87(7.8)$ & $71(13.0)$ & $60(12.0)$ & $0(0)$ \\
\hline 6 & $\begin{array}{l}\text { Port Transport } \\
\text { (TP1\&TP2)* }\end{array}$ & $68(6.1)$ & $62(11.3)$ & $60(12.0)$ & $0(0)$ \\
\hline 7 & $\begin{array}{l}\text { Market Area } \\
\text { (MA1\&MA2) * }\end{array}$ & $86(7.7)$ & $65(11.9)$ & $60(12.0)$ & $0(0)$ \\
\hline Total & & $1,105(100)$ & $546(49.4)$ & $500(91.6)$ & $8(1.6)$ \\
\hline
\end{tabular}

\section{Snail species and Morphometrics}

On the basis of shell morphology, $546(49.4 \%)$ of the snails collected were putatively identified as Biomphalaria pfeifferi, whereas 310(28.1\%), 101(9.1\%), and 147(13.3\%) were identified as Bulinus spp., Lymnaea and Bivalve. (Table 9). Other than Biomphalaria and Bulinus species (the intermediate host snails of $S$. mansoni and $S$. haematobium, respectively), the other commonly identified freshwater snail was Lymnaea natalensis. 
Table 9

Snail population and distribution numbers dead and live by habitat in Gorgora town, Western Dembia, Northwest Ethiopia 2020.

\begin{tabular}{|lllllll|}
\hline $\begin{array}{l}\text { Habitat } \\
\text { no. }\end{array}$ & Habitat name & $\begin{array}{l}\text { Biomphalaria } \\
\text { pfeifferi }\end{array}$ & $\begin{array}{l}\text { Bulinus } \\
\text { spp. }\end{array}$ & Lymnea & Bivalve & Total \\
\hline 1 & Deber Sina (DS) & 61 & 4 & 0 & 0 & 65 \\
\hline 2 & Tim \& Kim (TK) & 67 & 64 & 41 & 50 & 222 \\
\hline 3 & $\begin{array}{l}\text { Military Camp } \\
\text { 5th (MC) }\end{array}$ & 116 & 89 & 40 & 53 & 298 \\
\hline 4 & $\begin{array}{l}\text { Fish Farm } \\
\text { Center (FF) }\end{array}$ & 104 & 110 & 24 & 41 & 279 \\
\hline 5 & \begin{tabular}{l} 
Port Hotel (PH) \\
\hline 6
\end{tabular} & 71 & 16 & 0 & 0 & 87 \\
\hline 7 & $\begin{array}{l}\text { Port Transport } \\
\text { (TP) }\end{array}$ & 62 & 6 & 0 & 0 & 68 \\
\hline Total & $\begin{array}{l}\text { Market Area } \\
\text { (MA) }\end{array}$ & 65 & 21 & 0 & 0 & 86 \\
\hline
\end{tabular}

\section{Physico-chemical parameters of the water points}

During snail collection the average water temperature, $\mathrm{pH}$ and conductivity was recorded as indicated in Table 10 below

Table 10

Physico-chemical characteristics of snail collection site water at Lake Tana in Gorgora town, Western Dembia, Northwest Ethiopia 2020.

\begin{tabular}{|lllll|}
\hline Habitat no. & Habitat name & Temperature $\left({ }^{\circ} \mathrm{C}\right)$ & $\mathrm{pH}$ & Conductivity $(\boldsymbol{\mu S} / \mathrm{cm})$ \\
\hline 1 & Deber Sina (DS) & 21.2 & 9 & 204.26 \\
\hline 2 & Tim \& Kim (TK) & 25.4 & 8.3 & 126.01 \\
\hline 3 & Military Camp 5th (MC) & 24.5 & 9 & 200.06 \\
\hline 5 & Fish Farm Center (FF) & 24.2 & 9 & 195.16 \\
\hline 6 & Port Hotel (PH) & 25.7 & 7.84 & 129.08 \\
\hline 7 & Port Transport (TP) & 24.3 & 8.97 & 151.06 \\
\hline
\end{tabular}




\section{Discussion}

Schistosomiasis is the most prevalent helminthic infection in Ethiopia. Depending on various reasons, the prevalence of $S$. mansoni is ranging from less than $1 \%$ up to more than $90 \%$ in Ethiopia(9). In Western Dembia District, numerous studies conducted in different localities showed that the prevalence of $S$. mansoni were high among school-age children and preschool aged children. The present study was aimed to assess the distribution of snail and risk factors of $S$. mansoni in proximity to water contact points in Gorgora town.

The prevalence of intestinal schistosomiasis in the current study was $36.6 \%$ (Cl: $32-41.9)$. This result is higher when compared to studies carried out in Western Kenya 16.3\% (10), Kwale, 0.0\% (11), Western Uganda 27.8\%(12), Sesse Islands, Uganda 31.4\%(13), Nyanza, western Kenya 13\%(14), Chuahit, Northwest Ethiopia 11.2\%(15), Gorgora, Northwest Ethiopia 20.6\% (16), Hawassa, Southern Ethiopia 31\% (17), Zegie Peninsula, Northwestern Ethiopia 29.9\%(18), Jimma Zone, South West Ethiopia 27.6\% (19), Sanja, northwest Ethiopia 16.67\%(20), in Addiremets town, Western Tigray, Ethiopia,26.3\%(21).This difference may be because of ecological, climatic condition of study area, sample size used, contact with open water sources which is infested with infective stage of the parasites and presence of intermediate hosts in water bodies in the area or control activities. However, it was in line with another report from Sanja, Northwest Ethiopia 35\%(22). This similarity may be because of contact with open water sources.

The result of the present study was lower than previous studies conducted, in Mbita causeway, Western Kenya 45.1\%(23), Uganda 47.5\%(24), Mbita District, Western Kenya 76.8\%(25), Sanja, Northwest Ethiopia 83.3\%(26), Wolaita, Southern Ethiopia 81.3\%(27),Yachi, southwestern Ethiopia 42.9\%(28) Sanja, Northwest Ethiopia 5-9 and 10-14 years were $84.6 \%$ and $75.2 \%$, respectively (29), Similarly, the prevalence of $S$. mansoni infection in the present study was lower than the findings in Wondo Genet, Southern Ethiopia 74.9\%(30), Tigray, Ethiopia 42.4\%(31). Sanja, Northwest Ethiopia 89.9\%(32), Alamata, Northern Ethiopia 73.9\%(33), Hayk town, northeastern Ethiopia, 45\%(34). This variation might be due to the study area sociodemographic factors, ecological distribution of intermediate host (snail), local endemicity of the parasite, sample size, awareness regarding the transmission and prevention and difference in study period.

In the current study S. mansoni intensity of study participants was $30.5 \%, 27.0 \%$ and $42.6 \%$ for light, moderate, and heavy infection, respectively. The present study has high proportion of heavy intensity of infection, compared with a report from Western Kenya, (9.8\%)(10), Sanja, Northwest Ethiopia, (18.7\%) (32), Sanja, Northwest Ethiopia, (36.3\%)(26), and Addiremets town, Western Tigray, Ethiopia, (16.7\%) (21). Similarly, In Mbita District, Western Kenya, the intensity of infection was $16.1 \%$ heavy infections (25). In south Nyanza, western Kenya $12.6 \%$ had heavy intensity of infection (14). In Wondo Genet, Southern Ethiopia $42.4 \%$ (30) had high intensity of infection. This variation might be due to low level of education, poor hygienic practice and absence of control and preventive measures in the area. However, this finding was in contrast to a study carried out in Wolaita Zone, Southern Ethiopia, with an overall heavy intensity 
of infection of $56.4 \%$ (27). This variation might be due to infection rate and frequency of contact with cercaria contaminated water bodies.

In the current study S. mansoni was 7.7 times more likely to happen in study participants with the sometimes habit of contact to Lake Tana than those who had no habit of contact to Lake Tana. This is finding was positively associated when compared to studies carried out, in Western Kenya(10), higher than Wolaita Zone, Southern Ethiopia, (3.9 times) (27), while in Jimma Zone, South West Ethiopia children who have bathing habits in open water sources 8.8 times (19), southern Ethiopia, had shown that those who swum in the lake Hawassa are infected about 2.7 times more than those who do not(17), Sanja, northwest Ethiopia, had shown that those who swum in river about two times more than who do not(20), Jiga town, Northwest-Ethiopia, showed that habit of contact with river who were swum in river were 4.8 times(35). This variation might be due to immune status of participants, distance from the water source and frequency of contact with infected water which is a common scenario seen in almost all schistosomiasis is studies in the country and in the current study area.

S. mansoni was Ten times more likely to happen in participants with frequent habit of swimming in Lake Tana than those who had no habit of swimming. Frequent swimming had accounted for infection in a study carried out in Tigray, Ethiopia, (31), and Western Kenya (10). This is finding was in contrast to a report from Blantyre, Malawi where frequency of water contact was not significantly associated with Schistosoma infection (36). This variation might be due to time of water contact which is associated with shedding of infective $S$. mansoni cercaria.

The odds of getting S. mansoni infection among individuals Fishing and Farming was 7.9 times higher than those who do not. This is finding correlates with a report from western Kenya, where fishing in Lake Victoria was associated with a higher risk of infection(10).

With regard to proximity of water, the present study has demonstrated higher likely infection odds of $S$. mansoni infection among individuals living $1 \mathrm{~km}$ away from the shoreline; 1.7 times higher than those who living $1 \mathrm{~km}$ away from the shoreline at Lake Tana. This finding is supported by a study carried out in western Kenya where the school nearest the lake less than $1 \mathrm{~km}$ had a prevalence of $80 \%$ and the mean prevalence decreased to approximately $20 \%$ at a distance of $4 \mathrm{~km}$. In addition beyond the $4 \mathrm{~km}$ line, the prevalence decreased further to $2.8 \%$ for children living beyond $9 \mathrm{~km}$ from the shoreline (10). Similar finding in Kwale, Kenya, showed a significant low infection risk among participants who were residing far away from a river(11).

In Blantyre, Malawi, the odds of getting $S$. mansoni infection among individuals living less than $1 \mathrm{~km}$ was 5.3 times higher than $1 \mathrm{~km}$ living (36). This finding is not surprising as children who live closer to the lake are more likely to have contact with water on a regular basis (10).

The current study had revealed malacological diversity in the study area. As assessed using morphological methods, $546(49.4 \%)$ of the snails collected were putatively identified as B. pfeifferi, whereas 310(28.1\%), 101(9.1\%), and 147(13.3\%) were identified as Bulinus spp., Lymnaea and Bivalve, 
respectively. This result is consistent with the malacological survey conducted in freshwater bodies, in Sanja, Northwest Ethiopia, during January and April, where B. pfeifferi were 150(97.4\%) and 180(81.4\%), respectively and also B. forskalii and L. natalensis (29). In North-Western Nigeria, 788 (47.94\%) L. natalensis, 492 (29.93\%) B. trophicus and 364 (26.14\%) B. forskalii (37), were reported. This similarity might be due to the abundancy and occurrence of snail species were largely influenced by water quality.

Similarly, in Kpong Head Pond, Ghana a total of 1,034 snails were collected throughout the study period. Five different snail species were identified. The dominant schistosome vector species found were the $B$. truncates 735/1034 (71.0\%), followed by Biomphalaria 125/1034 (12.0\%) and B. globosus (6,1\%)(38). In Niger River Valley West Africa, $B$. truncatus was the most abundant species found, followed by $B$. forskalii, $R$. natalensis and $B$. pfeifferi that showed a significant positive association with dry season and a significant negative association with the wet season (39). In Mecha North West Ethiopia, $46.1 \%$ Helix, 23.7\% Lymnaea, 13.2\% Bulinus, 9.2\% Planorbin, and 7.9\% Oncomelania (40) was reported. In Middle Paranapanema River region of São Paulo, Brazil, of the 10,722 snails collected, $86.7 \%$ were in the Planorbidae family (75.5\% Biomphalaria and 11.2\% Drepanotrema) and $13.3 \%$ were other nonPlanorbidae species (Lymnaea, Melanoides, Physa and Pomacea) (41). This variation might be due to seasonality of snail abundance and freshwater snails are generally encountered in freshwater body that is polluted with human and animal excreta.

On the other hand, this finding was in contrast when compared to studies carried out in Kisumu City, western Kenya, which reported out of 1,059 snails collected, 407 (38.4\%) were putatively identified as $B$. sudanica,425(40.1\%) as B. pfeifferi and 227(21.5\%) as Bulinus globosus(42). In Mara River in Kenya and Tanzania, all the four different snail species were found to be attached to different aquatic weeds, with $B$. pfeifferi accounting for over half $(61.1 \%)$ of the snails attached to the sedge, followed by $B$. africanus and Lymnaea spp., accounting for $22.2 \%$ and $16.7 \%$, respectively(43). A study from Omo Gibe River Basin, southwest Ethiopia, reported distribution of snails as Bi. pfeifferi 2072(66.8\%), Bi. Sudanica 7(0.2\%), B. globosus 148(4.8\%), B. forskalii 133(4.3\%) and L. natalensis $747(24 \%)(44)$. The main reason for the variation here may be an increase in organic matter will increase the concentration of detritus and possibly the proliferation of algae that forms the diet of planorbid and prosobranch snails and prevalence of Schistosoma spp. infection, abundance, freshwater snails in the current study are showed that the ecology is suitable due to good vegetation cover, low alkaline $\mathrm{pH}$ and moderate temperature.

In the current study, the prevalence of schistosome infection in B. pfeifferi was $0.0 \%$. this finding is in line with a study conducted in Addiremets town, Western Tigray, Ethiopia, 0.0\%(21), North western Angola $0.0 \%(45)$, Sesse Islands, Uganda, $0.0 \%(13)$, Wolaita Zone, Southern Ethiopia $0.0 \%(27)$, Maksegnit and Enfranz Towns, northwestern Ethiopia, $0.0 \%$ (46), and Gombe or Bugamba $0.0 \%(47)$. This absence of infected snails may be associated with seasonality of infection with Schistosoma spp. In addition, the low prevalence of cercariae in this study might be due to the presence of other trematodes such as Echinostome cercariae and resistance of some snail species to trematode infection. 
On the other hand there are reports of snails shedding cercaria finding from a study conducted in Sanja, Northwest Ethiopia, $16.9 \%$ and $0.027 \%$ during February and April, respectively (29), Omo Gibe River Basin, southwest Ethiopia, 4.6\%(44), Hayk town, northeastern Ethiopia, from Ketie Stream, 3.2\%(34), Maksegnit and Enfranz Towns, northwestern Ethiopia, 14.3\% (46), western Tanzania Gombe National Park all col $12.3 \%$ of snails shed larvae (47), Niger River Valley West Africa 3.45\% (39), Kisumu City, western Kenya $1.7 \%$ in $B$. sudanica and $1.6 \%$ in B. pfeifferi (42). The reason for finding infected snails in these study areas in contrast to the current study could be the variation in season during sample collection.

\section{Conclusion}

In conclusion, high prevalence of S. mansoni infection was observed in Gorgora town. Swimming, fishing and farming were associated risk factors of contracting schistosomiasis. More than Eighty-two present of observed Schistosoma positive participant had proximity to lake water. A total of four different freshwater snail intermediate host species with a potential of transmitting diseases of human and veterinary public health importance had been identified. No Bi. pfefferi was found shedding cercariae. Health education, snail control and safe water supply is recommended.

\section{Abbreviations}

ArcGIS= Architecture Geographical Information System, $D A L Y S=$ Disability Adjusted Life Years, EPG= Eggs Per Gram, GIS= Geographical Information System, GPS= Global Positioning System, HH= House Holds, ICE = Information, Communication, and Education, LMIC = Low- and Middle-Income Countries, MDA= Mass Drug Administration, NTD= Neglected Tropical Disease, PC= Preventive Chemotherapy, PZQ= Praziquantel, SOP= Standard Operating Procedure, STH= Soil Transmitted Helimenth, WASH= WAter, Sanitation and Hygiene, WHO= World Health Organization

\section{Declarations}

\section{Ethics approval and consent to participate}

The proposed study was approved by the research and ethics committee University of Gondar, College of Medicine and Health Science ethical school of biomedical and laboratory science review committee who approved this work concerning the ethical issues.

\section{Ethical Considerations}

Ethical approval was obtained from the school of Biomedical and laboratory science (SBLS) ethical committee and a permission letter was obtained from Central Gondar Zonal Health Office, western Dembia District Health Bureau and selected study site Kebeles leaders. When the inhabitants of nearby villages in proxy to water sources are identified, the purpose, objectives and benefits of the study was explained. Consent and assent for participation was obtained from the children's parents/guardians. Study participant who was found positive for $S$. mansoni and other intestinal parasites infection was 
linked with the health center and treated with standard dose of anti-schstosomal and anti-helminthic treatment.

\section{Consent for publication}

Not applicable. This study does not contain any individual or personal data.

\section{Availability of data and materials}

Data is available upon request.

\section{Funding}

This research work was financed by University of Gondar, Ethiopia. The funder had no role in study design, data collection and analysis, decision to publish, or preparation of the manuscript.

\section{Authors' contributions}

$A A, M A, L W, A G, A D$ and $D A$, were involved in study conception, data collection and analysis, drafting the manuscript. AA, MA, AG and DA were critically reviewed the manuscript for intellectual content. All authors have read, edited and approved the manuscript.

\section{Acknowledgements}

Our sincere appreciation is extended to Mr. Getent Ayalew, Mr. Getayeneh Antehunegn, Mr. Amare Kiflie, Mr. Gezahegn Bewket, Mr. Tilahun Tegegn, Mr. Habtamu Asefa, Mr. Hailemaryam Getachew and Mr. Mesafint Eyayu for their enthusiastic and cooperative support in the preparation of Kato-Katz thick smear and GPS data analysis. We also appreciate Mrs. Wubit Mekonen (Urban Health Extention Nurse) for her commitment to administer the drug for the inhabitants who were found positive for intestinal parasites infection.

We gratefully acknowledge all study participants and Mr. Habetamu Asefa, Head of Gorgora Health Center, Gorgora Town, western Dembia District, Northwest Ethiopia for their participation and support during the data collection.

\section{Competing interests}

The authors declare that they have no competing interests.

\section{References}

1. Hotez PJ, Fenwick A, Savioli L, Molyneux DH. Rescuing the bottom billion through control of neglected tropical diseases. The Lancet. 2009;373(9674):1570-5. 
2. Organization WH. Prevention and control of schistosomiasis and soil-transmitted helminthiasis: report of a WHO expert committee. 2002.

3. Hussen S, Assegu D, Shimelis T. Prevalence of Schistosoma mansoni infection in Ethiopia: A systematic review and meta-analysis. BioRxiv. 2019:610113.

4. Anderson R, May R. Prevalence of schistosome infections within molluscan populations: observed patterns and theoretical predictions. Parasitology. 1979;79(1):63-94.

5. WHO. A filed guide to African freshwater snails 1987.

6. Mello D. A comparative morphology of the genital system of some African species of Biomphalaria (Mollusca, Planorbidae). Revista Brasileira de Biologia. 1972;32:442-50.

7. Ndione RA, Bakhoum S, Haggerty C, Jouanard N, Senghor S, Ndao PD, et al. Intermediate Host Snails of Human Schistosomes in the Senegal River Delta: Spatial Distribution According to Physicochemical Parameters. Invertebrates-Ecophysiology and Management: IntechOpen; 2019.

8. Kato K. Comparative examinations. Jpn J Parasitol. 1954;3:35.

9. Haidar J, HaileMariam D, Demisse T, Kloos H. The Epidemiology and Ecology of Health and Disease in Ethiopia. Addis Ababa, Ethiopia: Shama Books; 2006.

10. Handzel T, Karanja DM, Addiss DG, Hightower AW, Rosen DH, Colley DG, et al. Geographic distribution of schistosomiasis and soil-transmitted helminths in Western Kenya: implications for anthelminthic mass treatment. The American journal of tropical medicine and hygiene. 2003;69(3):318-23.

11. Chadeka EA, Nagi S, Sunahara T, Cheruiyot NB, Bahati F, Ozeki Y, et al. Spatial distribution and risk factors of Schistosoma haematobium and hookworm infections among schoolchildren in Kwale, Kenya. PLoS neglected tropical diseases. 2017;11(9):e0005872.

12. John R, Ezekiel M, Philbert C, Andrew A. Schistosomiasis transmission at high altitude crater lakes in Western Uganda. BMC infectious Diseases. 2008;8(1):110.

13. Standley CJ, Adriko M, Arinaitwe M, Atuhaire A, Kazibwe F, Fenwick A, et al. Epidemiology and control of intestinal schistosomiasis on the Sesse Islands, Uganda: integrating malacology and parasitology to tailor local treatment recommendations. Parasites \& vectors. 2010;3(1):64.

14. Sang HC, Muchiri G, Ombok M, Odiere MR, Mwinzi PN. Schistosoma haematobium hotspots in south Nyanza, western Kenya: prevalence, distribution and co-endemicity with Schistosoma mansoni and soil-transmitted helminths. Parasites \& vectors. 2014;7(1):125.

15. Alemu A, Tegegne Y, Damte D, Melku M. Schistosoma mansoni and soil-transmitted helminths among preschool-aged children in Chuahit, Dembia district, Northwest Ethiopia: prevalence, intensity of infection and associated risk factors. BMC Public Health. 2016;16(1):422.

16. Essa T, Birhane Y, Endris M, Moges A, Moges F. Current status of Schistosoma mansoni infections and associated risk factors among students in Gorgora town, Northwest Ethiopia. ISRN Infectious Diseases. 2012;2013.

17. Tadege B, Shimelis T. Infections with Schistosoma mansoni and geohelminths among school children dwelling along the shore of the Lake Hawassa, southern Ethiopia. PloS one. 
2017;12(7):e0181547.

18. Abdi M, Nibret E, Munshea A. Prevalence of intestinal helminthic infections and malnutrition among schoolchildren of the Zegie Peninsula, northwestern Ethiopia. Journal of infection and public health. 2017; 10(1):84-92.

19. Bajiro M, Tesfaye S. Schistosoma mansoni infection prevalence and associated determinant factors among school children in Mana District, Jimma Zone, Oromia Region, South west Ethiopia. J bacteriol \&parasitol. 2017;8(5):329.

20. Andargie AA, Abera AS. Determinants of Schistosoma mansoni in Sanja health center, north West Ethiopia. BMC public health. 2018;18(1):620.

21. Gebreyohanns A, Legese MH, Wolde M, Leta G, Tasew G. Prevalence of intestinal parasites versus knowledge, attitude and practices (KAPs) with special emphasis to Schistosoma mansoni among individuals who have river water contact in Addiremets town, Western Tigray, Ethiopia. PloS one. 2018;13(9):e0204259.

22. Woldegerima E, Bayih AG, Tegegne Y, Aemero M, Jejaw Zeleke A. Prevalence and Reinfection Rates of Schistosoma mansoni and Praziquantel Efficacy against the Parasite among Primary School Children in Sanja Town, Northwest Ethiopia. Journal of parasitology research. 2019;2019.

23. Chadeka EA, Nagi S, Cheruiyot NB, Bahati F, Sunahara T, Njenga SM, et al. A high-intensity cluster of Schistosoma mansoni infection around Mbita causeway, western Kenya: a confirmatory crosssectional survey. Tropical medicine and health. 2019;47(1):1-4.

24. Kabatereine NB, Brooker S, Tukahebwa EM, Kazibwe F, Onapa AW. Epidemiology and geography of Schistosoma mansoni in Uganda: implications for planning control. Tropical medicine \& international health : TM \& IH. 2004;9(3):372-80.

25. Nagi S, Chadeka EA, Sunahara T, Mutungi F, Justin YKD, Kaneko S, et al. Risk factors and spatial distribution of Schistosoma mansoni infection among primary school children in Mbita District, Western Kenya. PLoS Negl Trop Dis. 2014;8(7):e2991.

26. Tesfie A, Getnet G, Abere A, Yihenew G, Belete Y, Kassa M, et al. Praziquantel is an effective drug for the treatment of Schistosoma Mansoni infection among school-aged children in Northwest Ethiopia. Tropical Medicine and Health. 2020;48(1):1-8.

27. Alemayehu B, Tomass Z. Schistosoma mansoni infection prevalence and associated risk factors among schoolchildren in Demba Girara, Damot Woide District of Wolaita Zone, Southern Ethiopia. Asian Pacific journal of tropical medicine. 2015;8(6):457-63.

28. Bekana T, Hu W, Liang S, Erko B. Transmission of Schistosoma mansoni in Yachi areas, southwestern Ethiopia: new foci. Infectious diseases of poverty. 2019;8(1):1.

29. Alebie G, Erko B, Aemero M, Petros B. Epidemiological study on Schistosoma mansoni infection in Sanja area, Amhara region, Ethiopia. Parasites \& vectors. 2014;7(1):15.

30. Erko B, Degarege A, Tadesse K, Mathiwos A, Legesse M. Efficacy and side effects of praziquantel in the treatment of Schistosomiasis mansoni in schoolchildren in Shesha Kekele Elementary School, Wondo Genet, Southern Ethiopia. Asian Pacific journal of tropical biomedicine. 2012;2(3):235-9. 
31. Desta H, Bugssa G, Demtsu B. The current status of Schistosoma mansoni infection among school children around Hizaty Wedicheber Microdam in Merebmieti, Ethiopia. Journal of Bacteriology \& Parasitology. 2014;5(5):1.

32. Worku L, Damte D, Endris M, Tesfa H, Aemero M. Schistosoma mansoni infection and associated determinant factors among school children in Sanja Town, Northwest Ethiopia. Journal of parasitology research. 2014;2014.

33. Abebe N, Erko B, Medhin G, Berhe N. Clinico-epidemiological study of Schistosomiasis mansoni in Waja-Timuga, District of Alamata, northern Ethiopia. Parasites \& vectors. 2014;7(1):158.

34. Amsalu G, Mekonnen Z, Erko B. A new focus of schistosomiasis mansoni in Hayk town, northeastern Ethiopia. BMC Research Notes. 2015;8(1):1-6.

35. Wubet K, Damtie D. Prevalence of Schistosoma mansoni Infection and Associated Risk Factors among School Children in Jiga Town, Northwest-Ethiopia: A Cross-Sectional Study. Journal of Parasitology Research. 2020;2020.

36. Kapito-Tembo AP, Mwapasa V, Meshnick SR, Samanyika Y, Banda D, Bowie C, et al. Prevalence distribution and risk factors for Schistosoma hematobium infection among school children in Blantyre, Malawi. PLoS Negl Trop Dis. 2009;3(1):e361.

37. Auta T, Alkali E, Michael EA. Population Dynamics, Diversity and Distribution of Freshwater Snails in Zobe Dam, Dutsin-Ma, North-Western Nigeria. Asian Journal of Environment \& Ecology. 2018:1-7.

38. Yirenya-Tawiah D, Rashid AA, Futagbi G, Aboagye I, Dade M. Prevalence of snail vectors of schistosomiasis in the Kpong Head Pond, Ghana. West African Journal of Applied Ecology. 2011;18:39-45.

39. Rabone M, Wiethase JH, Allan F, Gouvras AN, Pennance T, Hamidou AA, et al. Freshwater snails of biomedical importance in the Niger River Valley: evidence of temporal and spatial patterns in abundance, distribution and infection with Schistosoma spp. Parasites \& vectors. 2019;12(1):498.

40. Salew D, Munshea A. Identification and distribution of intermediate host snails in Mecha woreda, Amhara, North West of Ethiopia. Biodiversity Int J. 2018;2(5):441-5.

41. Palasio RGS, Xavier IG, Chiaravalotti-Neto F, Tuan R. Diversity of Biomphalaria spp. freshwater snails and associated mollusks in areas with schistosomiasis risk, using molecular and spatial analysis tools. Biota Neotropica. 2019;19(4).

42. Opisa S, Odiere MR, Jura WG, Karanja DM, Mwinzi PN. Malacological survey and geographical distribution of vector snails for schistosomiasis within informal settlements of Kisumu City, western Kenya. Parasites \& vectors. 2011;4(1):226.

43. Dida GO, Gelder FB, Anyona DN, Matano A-S, Abuom PO, Adoka SO, et al. Distribution and abundance of schistosomiasis and fascioliasis host snails along the Mara River in Kenya and Tanzania. Infection Ecology \& Epidemiology. 2014;4(1):24281.

44. Mereta ST, Bedewi J, Yewhalaw D, Mandefro B, Abdie Y, Tegegne D, et al. Environmental determinants of distribution of freshwater snails and trematode infection in the Omo Gibe River Basin, southwest Ethiopia. Infectious diseases of poverty. 2019;8(1):93. 
45. Allan F, Sousa-Figueiredo JC, Emery AM, Paulo R, Mirante C, Sebastião A, et al. Mapping freshwater snails in north-western Angola: distribution, identity and molecular diversity of medically important taxa. Parasites \& vectors. 2017;10(1):460.

46. Gashaw F, Aemero M, Legesse M, Petros B, Teklehaimanot T, Medhin G, et al. Prevalence of intestinal helminth infection among school children in Maksegnit and Enfranz Towns, northwestern Ethiopia, with emphasis on Schistosoma mansoni infection. Parasites \& vectors. 2015;8(1):567.

47. Bakuza JS, Gillespie R, Nkwengulila G, Adam A, Kilbride E, Mable BK. Assessing S. mansoni prevalence in Biomphalaria snails in the Gombe ecosystem of western Tanzania: the importance of DNA sequence data for clarifying species identification. Parasites \& vectors. 2017;10(1):584.

\section{Figure Note}

Figure 1 and Figure 2 are not available with this version.

\section{Figures}

\section{Image not available with this version}

Figure 1

\section{Image not available with this version}

Figure 1 


\section{Image not available with this version}

Figure 2

\section{Image not available with this version}

Figure 2

Spatial distribution of schistosomiasis-infected inhabitants' participants and Snail in Lake Tana to Gorgora

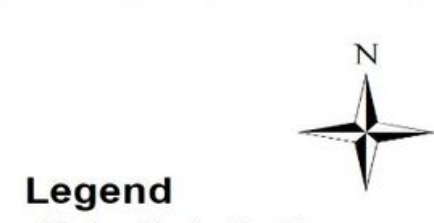

Kato-Katz finding

- No

- Yes

I Snail

- Institution

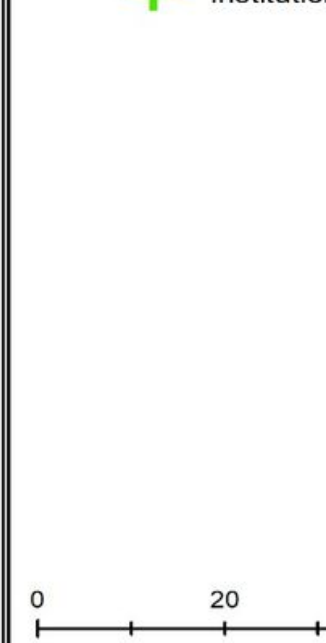

Figure 3 
Spatial distribution S. mansoni infection and snail survey sites the shoreline of Lake Tana in Gorgora town, Western Dembia, Northwest Ethiopia 2020. Note: The designations employed and the presentation of the material on this map do not imply the expression of any opinion whatsoever on the part of Research Square concerning the legal status of any country, territory, city or area or of its authorities, or concerning the delimitation of its frontiers or boundaries. This map has been provided by the authors.

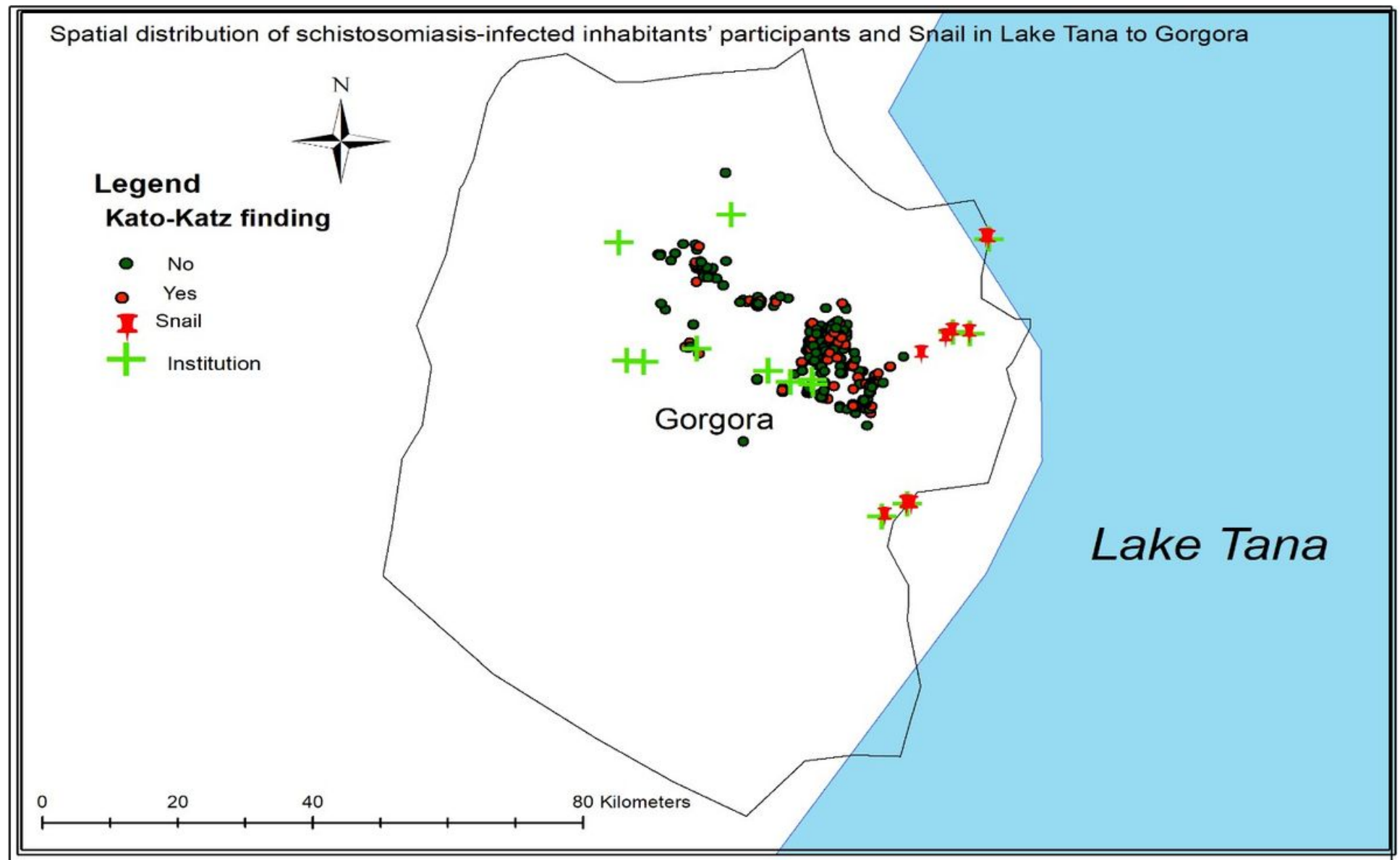

\section{Figure 3}

Spatial distribution S. mansoni infection and snail survey sites the shoreline of Lake Tana in Gorgora town, Western Dembia, Northwest Ethiopia 2020. Note: The designations employed and the presentation of the material on this map do not imply the expression of any opinion whatsoever on the part of Research Square concerning the legal status of any country, territory, city or area or of its authorities, or concerning the delimitation of its frontiers or boundaries. This map has been provided by the authors. 

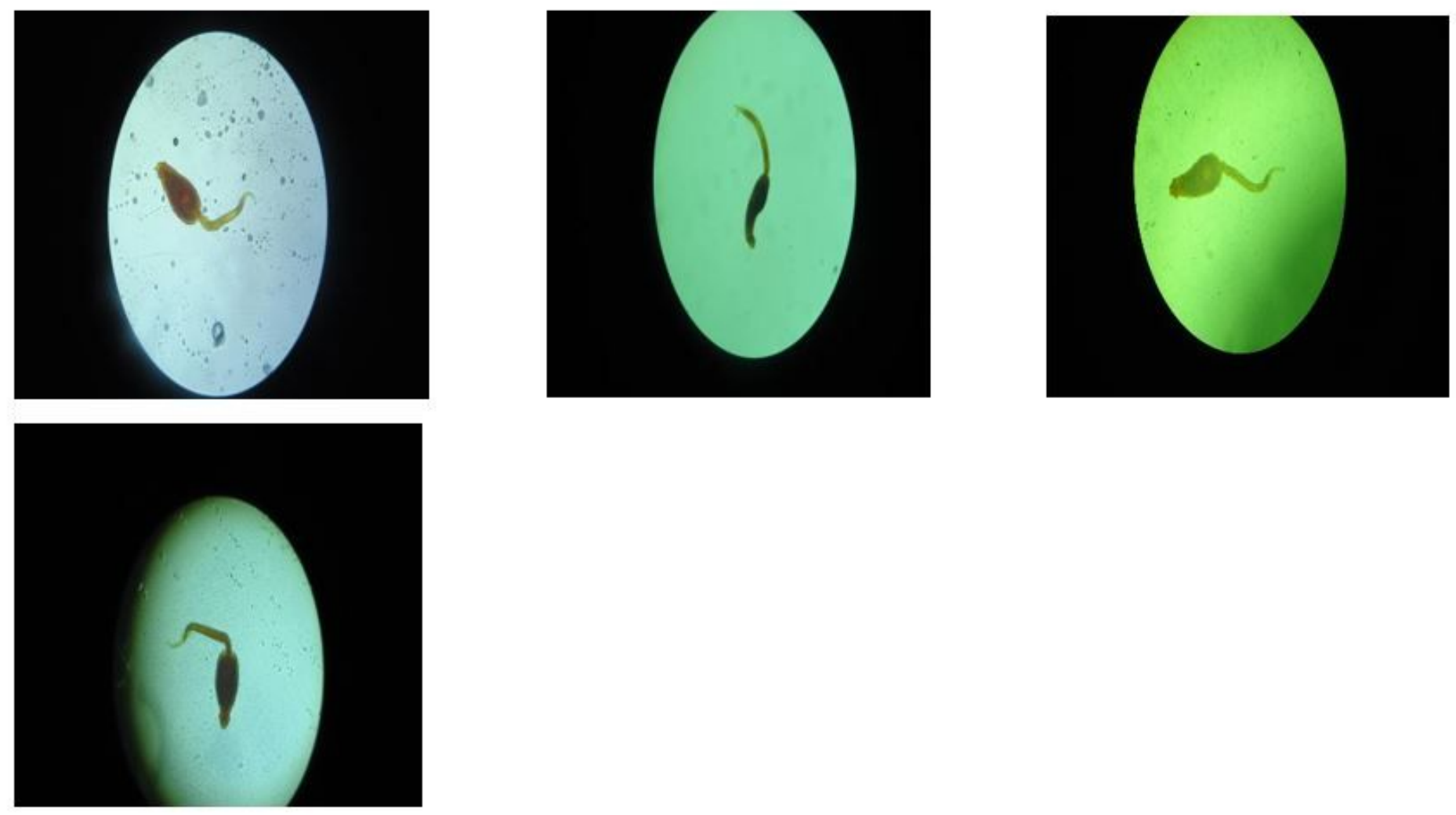

Figure 4

Cercariae that emerged from Biomphalaria pfeifferi in Lake Tana, in Gorgora town, Western Dembia, Northwest Ethiopia 2020.
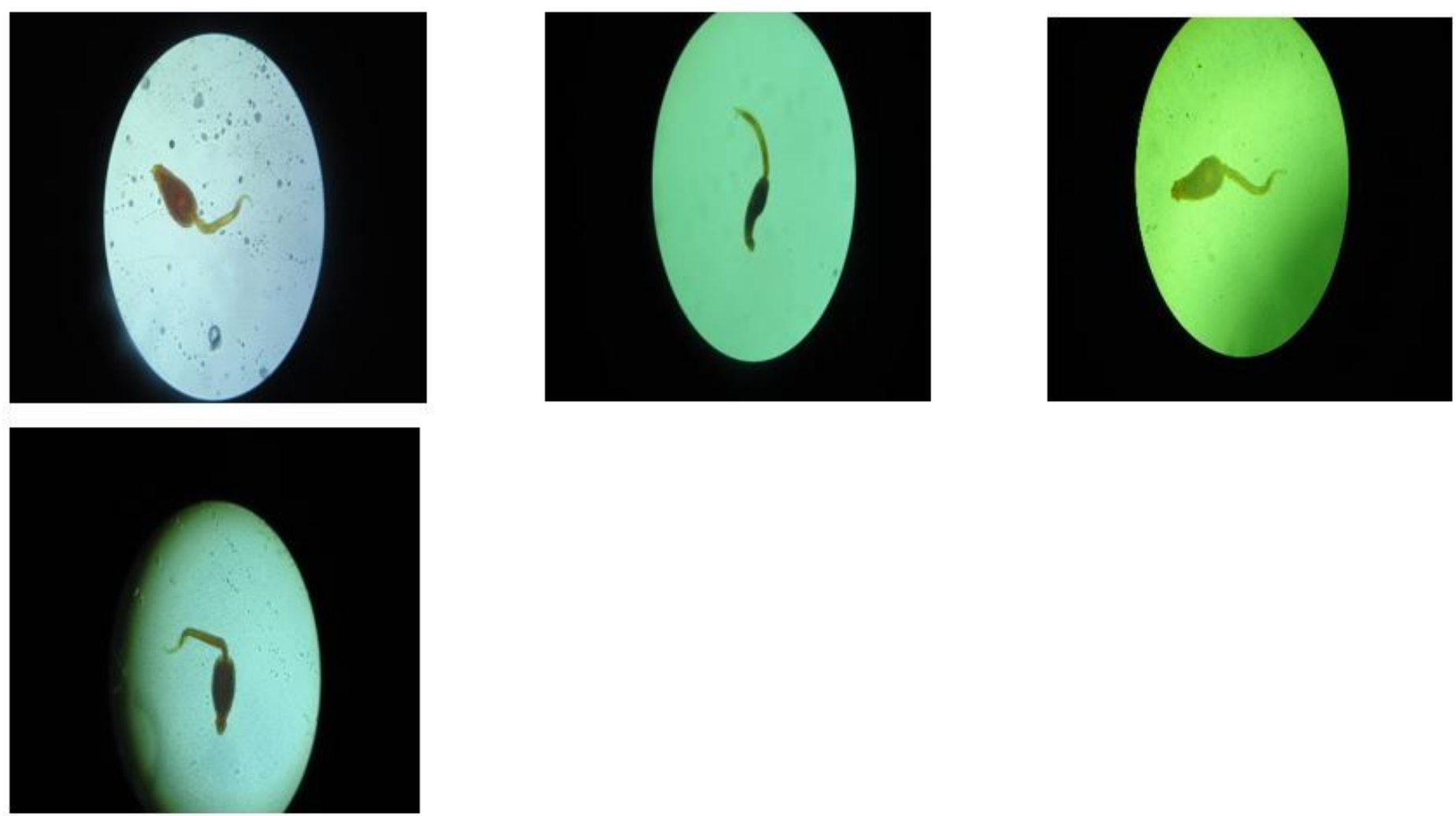
Figure 4

Cercariae that emerged from Biomphalaria pfeifferi in Lake Tana, in Gorgora town, Western Dembia, Northwest Ethiopia 2020.

\section{Supplementary Files}

This is a list of supplementary files associated with this preprint. Click to download.

- IMG1533.JPG

- IMG1533.JPG 